\title{
Clinical Usefulness of Immunostaining with CEA in Distinction between Malignant Melanoma and Spitz Nevus
}

\author{
Johno T, Egawa K, Jinnin M, Fukushima S, Masuguchi S, Inoue Y, Ihn H*
}

Department of Dermatology \& Plastic Surgery, Faculty of Life Sciences, Kumamoto University, Kumamoto, Japan

*Corresponding author: Prof. Hironobu Ihn, MD, PhD, Department of Dermatology and Plastic Surgery, Faculty of Life Sciences, Kumamoto University, 1-1-1 Honjo, Kumamoto 860-8556, Japan, Tel: + 81-96-373-5233/ Fax: + 81-96-373-5235; E-mail: ihn-der@kumamoto-u.ac.jp

\begin{abstract}
Background: Malignant melanoma (MM) is one of the most fatal cancers in the field of dermatology. On the other hand, Spitz Nevi (SN) is a benign tumor of melanocytes. Prognosis of these neoplastic tumors is completely different, but their histopathological findings are very similar. CEA (carcinoembryonic antigen) is one of the most familiar tumor markers in colon cancer. But it has been found to be expressed in many diseases and various tissues. In human skin, CEA is expressed in sebaceous glands and sweat glands.

Objectives: The aim of this study is to investigate the expression of CEA in MM and SN.

Materials and Methods: Six-teen tissues of MM and Eleven tissues of SN were immunohistochemically stained with polyclonal antibody against CEA.

Results: Strong positive staining was observed in MMs, on the other hand, we could not observe strong staining in SN. We suggest that immunohistochemical staining of CEA may distinguish MM from SN.
\end{abstract}

Keywords: Immunohistochemistry; Antigen-retrieval; Diagnosis
Received date: November 21, 2015

Accepted date: January 25, 2016

Published date: January 29, 2016

Citation: Ihn, H., et al. Clinical Usefulness of Immunostaining with CEA in Distinction between Malignant Melanoma and Spitz Nevus. (2016) Invest Dermatol Venereol Res 1(2): 40- 43.

DOI: $10.15436 / 2381-0858.16 .013$

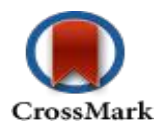

\section{Introduction}

Malignant melanoma (MM) is the most famous and the most fatal cancer in the field of dermatology. Skin lesions suspicious for melanoma typically meet one or more of the "ABCDE" criteria (Asymmetry, Border, Color, Diameter and Elevation) ${ }^{[1,2]}$. Then, clinicians have to resect broadly, and have to diagnose pathologically. But it is often difficult to distinguish MM from other melanocytic diseases pathologically. Therefore, many stainings have been developed to identify MMs. MM cells are positive for HMB-45, S-100 protein, MART-1 and so on ${ }^{[3-5]}$.

Spitz nevus (SN) was first described by Sophie Spitz in $1948^{[6]}$. She originally called it "benign juvenile melanoma". Now, "Spitz nevus" is the more commonly used term for benign juvenile melanoma because it is also encountered in adults and the term"melanoma" carries a negative connotation. The SN lesions can be asymptomatic or have a history of rapid, but limited growth. Grossly, classical SN are well circumscribed, symmetrical, small to medium sized firm papules, approximately 3-10mm in size, with smooth discrete borders and a uniform color, which is typically pink or flesh colored. SN can occur in various shapes, such as flat and polypoid. SN usually is found on the face, neck, or lower extremities, but can occur anywhere on the body. Histologically, the classical SN consists of large spindle and/or epithelioid melanocytes arrayed as epidermal nests grouped in a vertical orientation, so called "bunches of bananas" or "raining down pattern", with clefting artifact at the perimeter. The nests are fairly uniform, nonconfluent, and evenly spaced. There is little or no Pagetoid spread pattern. Epidermal changes include acanthosis, hypergranulosis, and hyperkeratosis. The intradermal pattern displays maturation, with single-file or single unit arrays descending to the base. Eosinophilic Kamino bodies are frequently found along the dermoepidermal junction. Kamino bodies are globular clusters which represent apoptotic, degenerative melanocytes. At the dermal base, there is no mitoses, no pushing deep margins, and lack 
of significant pleomorphism ${ }^{[7]}$. The defferential diagnosis of SN includes MM. These features are insufficient for discriminating SN from MM. Furthermore, SN cells are also stained positive with immunostainings which are positive for MM.

Prognosis of these neoplastic tumors is completely different, but their histopathological findings are very similar. It is needed to find the way to distinguish MM from SN clearly and simply.

CEA (carcinoembryonic antigen) is one of the most familiar tumor markers in colon cancer. It has been known that CEA has many subgroups, and works in connection with cell adherence, tumor immunity and so on ${ }^{[8]}$. However, anti-polyclonal-CEA antibody (poly-CEA) which reacts with almost all human CEA family as an antigen is only used in immunostaining. It has been found to be expressed in many diseases and various tissues. In human skin, CEA is expressed in sebaceous glands and sweat glands ${ }^{[9]}$. In 1993, Sanders et al. reported that MMs were positive for poly-CEA in immnostaining ${ }^{[10]}$. But, it has not used so much in clinical areas.

In this report, we investigated the poly-CEA immunoreactivity for $\mathrm{MM}$ and $\mathrm{SN}$, and clarity its usefulness to distinguish MM from SN.

\section{Materials and Methods}

\section{Tissues}

The surgically resected specimens were used for this study. They included 16 of MM: 12 primary lesions, 2 LN metastatic lesions and 2 skin metastatic lesions, and 11 of SN. All specimens were diagnosed by clinical and histopathological findings already known. All samples were fixed in neutral buffered formalin, embedded in paraffin, and prepared for hematoxylin-eosin examination.

\section{Immunohistochemical stainings}

Immunohistochemical staining on paraffin-embedded section was performed using the standard streptavidin-biotin-peroxidase complex method with VECTASTAIN Elite ABC Kit. Four $\mu \mathrm{m}$ thick sections were mounted on silane-coated slide (Dako), then deparaffinized with xylene and rehydrated through a graded series of ethyl alcohol and PBS. Next, antigen-retrieval was performed using microwave for 5 minutes. After washing in PBS, non-specific binding sites were blocked with $10 \%$ normal rabbit serum at room temperature for 1 hour. Excess serum was removed from the sections. Then, the samples were incubated with the primary antibody at $4^{\circ} \mathrm{C}$ overnight. Following washing with PBS, they were incubated with biotinylated rabbit-anti goat $\mathrm{IgG}$ for 30 minutes at room temperature. The slides were rinsed and incubated with the avidin/biotin complex at room temperature for 1 hour. Visualization of the peroxidase reaction was achieved with diaminobenzidine (DAB), followed by counterstaining with Giemsa for decolorizing melanin.

\section{Results}

At first, we reconfirmed the result of general staining for melanocytic lesions, HMB-45, S-100 protein and MART-1. Positive staining for HMB-45, S-100 protein and melan A was observed in all MMs and SNs (Table1).
Table 1: Immunohistochemical staining of S - 100, HMB - 45, MART 1 in Maligant lymphoma and Spitz nevus.

\begin{tabular}{|l|c|c|c|c|c|c|}
\hline \multirow{2}{*}{} & \multicolumn{2}{|c|}{ S - 100 } & \multicolumn{2}{c|}{ HMB - 45 } & \multicolumn{2}{c|}{ MART - 1 } \\
\cline { 2 - 7 } & - & + & - & + & - & + \\
\hline Maligant Melanoma & \multicolumn{7}{|c|}{} \\
\hline Primary (n = 12) & 0 & 12 & 0 & 12 & 0 & 12 \\
\hline LN meta (n = 2) & 0 & 2 & 0 & 2 & 0 & 2 \\
\hline Skin meta (n= 2) & 0 & 2 & 0 & 2 & 0 & 2 \\
\hline Spitz nevus (n= 11) & 0 & 11 & 0 & 11 & 0 & 11 \\
\hline
\end{tabular}

S - 100, HMB - 45 and MART - 1 are general staining for MM and other melanocytic lesion. All of MM and $\mathrm{SN}$ sections are stained positive for each.

Next, we found strong positive staining for poly-CEA in all MMs included metastatic lesions. In 12 primary lesions, 5 cases were,++ 7 cases were + . In 4 metastatic lesions, we could see all positive poly-CEA staining much stronger (Figure 1 - 3). At least we would suggest that specimens positive for poly-CEA have the possibility of MM. Previous reports indicated that strong positive staining for poly-CEA was observed in MM specimens, similar to our result.

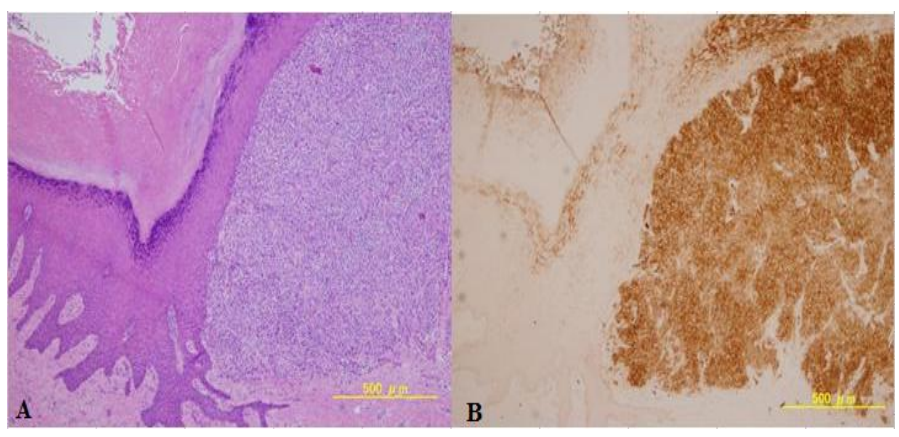

Figure 1: Malignant melanoma primary lesion.

H.E staining of MM primary lesion (A). Tumor cells exist in upper dermis. CEA staining of the same section (B). Tumor cells are stained very strongly.

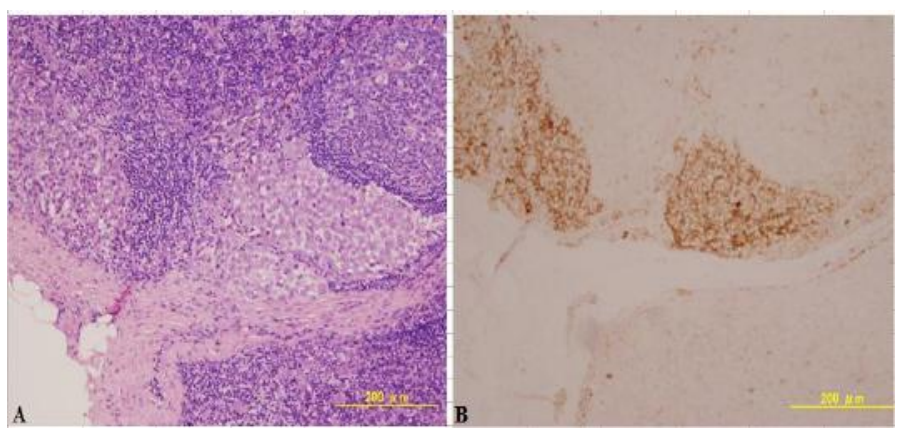

Figure 2: Malignant melanoma lymph nodes metastasis lesion. H.E staining of MM metastatic lesion in lymph nodes (A). Tumor cells exist in peripheral zone of lymph nodes. CEA staining of the same sections (B). Tumor cells are stained very strongly. 


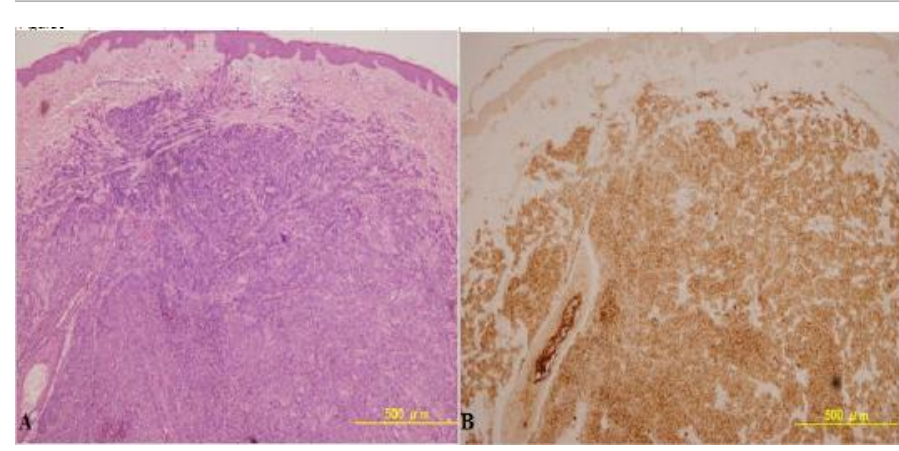

Figure 3: Malignant melanoma skin metastasis lesion.

H.E staining of MM metastatic lesion in skin (A). Tumor cells exist in dermis. CEA staining of the same sections (B). Tumor cells are stained very strongly.

On the other hand, we could observe little poly-CEA staining in SN. One specimen of SN showed a little positive staining for poly-CEA, but the others were almost negative (4 cases,- 6 cases \pm ) (Figure 4, Table 2). In any cases, the density of staining was all intentionally weaker compared with MM.

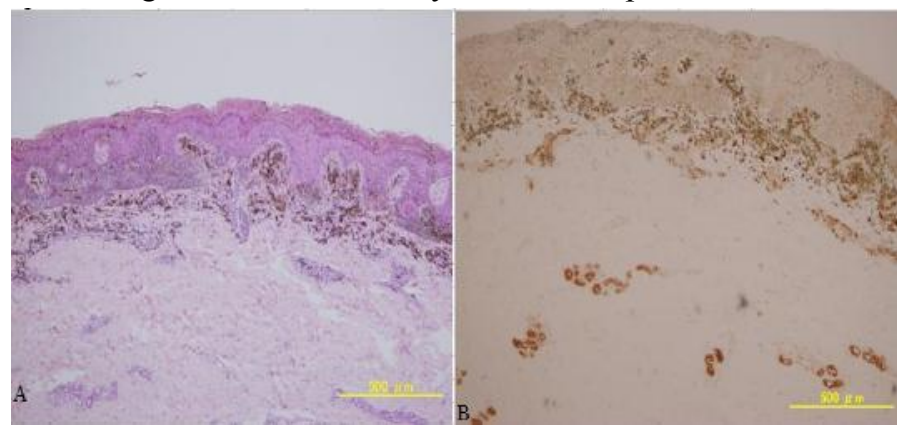

Figure 4: H.E staining of Spitz nevus (A). Tumor cells exist in upper dermis with melanin.CEA staining of the same section (B). Tumor cells are stained weaker than sweat glands in lower dermis. Melanin in tumor cells which was decolored by Giemsa staining looks bluish brown.

Table 2: Immunohistochemical staining of poly-CEA in Maligant lymphoma and Spitz nevus.

\begin{tabular}{|l|c|c|c|c|}
\hline \multirow{2}{*}{} & \multicolumn{4}{|c|}{ poly-CEA } \\
\cline { 2 - 5 } & - & \pm & + & ++ \\
\hline Maligant Melanoma & \multicolumn{4}{|c|}{} \\
\hline Primary (n= 12) & 0 & 0 & 7 & 5 \\
\hline LN meta (n= 2) & 0 & 0 & 1 & 1 \\
\hline Skin meta (n= 2) & 0 & 0 & 0 & 2 \\
\hline Spitz nevus (n= 11) & 4 & 6 & 1 & 0 \\
\hline
\end{tabular}

The result of poly-CEA staining after antiagen-retrieval -: not visible, 土: few cells stained weakly positive. +: positive clearly. ++ : strongly positive

Gambichler et al. suggested that expression of CEACAM1 in MM is an independent factor for metastasis risk ${ }^{[11]}$. We found that the frequency of SN positive for poly-CEA was much less than that of MMs. This suggests that MM have capability of metastasis, but SN do not.

\section{Discussion}

$\mathrm{MM}$ is a serious form of skin cancer, and the prevalence of MM annually is increasing faster than that of any other cancers. In Caucasians, exposure to the sunlight may be an important causal factor of MM. On the other hand, in more heavily pigmented races, many of MM occurs on the skin of the unexposed soles. MM may be divided into four clinical types: lentigo maligna melanoma, superficial spreading melanoma, nodular melanoma, and acral lentiginous melanoma. In Eastern Asia including Japan, acral lentiginous type occurr more frequently than any other types ${ }^{[11,12]}$.

The 10-years survival rate in the early stage of $\mathrm{MM}$ is over $80 \%$. But, the patients with MM in stage III B and stage IV have an unfavorable outcome, with 10 -year survivals of less than $50 \%$ and less than $10 \%$, respectively ${ }^{[12]}$. Therefore, we have to find and treat MM as early as possible.

Although the 'gold standard' for melanoma diagnosis remains histopathological analysis the dermoscopists play a significant role in the diagnostic process at present. But the judgement is sometimes difficult. Therefore, many researchers have investigated the diagnostic methods of MM.

The recent studies have shown that melanomas differ from nevi by the presence of frequent genes or losses of particular chromosomal regions, for example 6p25-RREB1, 6q23Myb, 11q13-CCND1 and so on ${ }^{[13-16]}$. We will be able to detect melanomas using a multi-colored fluorescence in situ hybridization (FISH) which detects copy number changes of chromosomal regions commonly found to be aberrant in melanoma. Takata et al. showed clearly that MM essentially differs from the SN in respect of variation or deficiencies, such as RAS, BRAF and CDKN2A genes ${ }^{[17]}$. Kozubek et al. reported that the miRNA profiling could distinguish MM from nevi ${ }^{[18]}$.

\section{Conclusion}

It is thought that the judgment of MM and SN would be possible by these techniques. But these new methods are not common and not available in general clinical laboratory.

On the other hand, poly-CEA is one of the most available antibody. Moreover, this antigen-retrieval method and staining method are also simple. Limitations of our study include the absence of functional studies. But, our data may give supports to distinguish MM from SN clearly and simply.

A small number of the patients with MM and those with SN were included in these studies. Further studies are required to clarify the clinical usefulness of immunohistochemical staining of CEA that may distinguish MM from SN. 


\section{References}

1. Abbasi, N.R., Shaw, H.M., Rigel, D.S., et al. Early diagnosis of cutaneous melanoma: Revisiting the ABCD criteria. (2004) JAMA 292(22): 2771-2776.

2. Strayer, S.M., Reynolds, P.L. Diagnosing skin malignancy: Assessment of predictive clinical criteria and risk factors. (2003) J Fam Pract 52(3): 210-218.

3. Wick, M.R., Swanson, P.E., Rocamora, A. Recognition of malignant melanoma by monoclonal antibody HMB-45. An immunohistochemical study of 200 paraffin-embedded cutaneous tumors. (1988) J Cutan Pathol 15(4): 201-207.

4. Nakajima, T., Watanabe, S., Sato, Y., et al. Immunohistochemical demonstration of S 100 protein in malignant melanoma and pigmented nevus, and its diagnostic application. (1982) Cancer 50(5): 912-918.

5. Fetsch, P.A., Cormier, J., Hijazi, Y.M. Immunocytochemical detection of MART-1 in fresh and paraffin-embedded malignant melanomas. (1997) J Immunother 20(1): 60-64.

6.Spitz, S. Melanomas of childhood. (1948) Am J Pathol 24(3): 591609.

7. Miteva, M., Lazova, R. Spitz Nevus and Atypical Spitzoid Neoplasm. (2010) Semin Cutan Med Surg 29(3): 165-173.

8. Gambichler, T., Grothe, S., Rotterdam, S., et al. Protein expression of carcinoembryonic antigen cell adhesion molecules in benign and malignant melanocytic skin lesions. (2009) Am J Clin Pathol 131(6): 782-787.

9. Egawa, K., Honda, Y., Ono, T., et al. Immunohistochemical demonstration of carcinoembryonic antigen and related antigens in various cutaneous keratinous neoplasms and verruca vulgaris. (1998) Br J Dermatol 139(2): 178-185.
10. Sanders, D.S., Evans, A.T., Allen, C.A., et al. Classification of CEA-related positivity in primary and metastatic malignant melanoma. (1994) J Pathol 172(4): 343-348.

11. Kukita, A., Ishihara, K. Clinical features and distribution of malignant melanoma and pigmented nevi on the soles of the feet in Japan. (1989) J Invest Dermatol 92(5 Suppl): 210S-213S.

12. Ishihara, K., Saida, T., Yamamoto, A. Updated statistical data for malignant melanoma in Japan. (2001) Int J Clin Oncol 6(3): 109-116.

13. Bauer, J., Bastian, B.C. Distinguishing melanocytic nevi from melanoma by DNA copy number changes: comparative genomic hybridization as a research and diagnostic tool. (2006) Dermatol Ther 19(1): 40-49.

14. Boi, S., Leonardi, E., Fasanella, S., et al. The four-color FISH probe in the diagnosis of melanocytic lesions. (2010) J Eur Acad Dermatol Venereol 24(10): 1235-1236.

15. Newman, M.D., Lertsburapa , T., Mirzabeigi, M., et al. Fluorescence in situ hybridization as a tool for microstaging in malignant melanoma. (2009) Mod Pathol 22(8): 989-995.

16. Busam, K.J., Fang, Y., Jhanwar, S.C., et al. Distinction of conjunctival melanocytic nevi from melanomas by fluorescence in situ hybridization. (2010) J Cutan Pathol 37(2): 196-203.

17. Takata, M., Lin, J., Takayanagi, S., et al. Genetic and epigenetic alterations in the differential diagnosis of malignant melanoma and spitzoid lesion. (2007) Br J Dermatol 156(6): 1287-1294.

18. Kozubek, J., Ma, Z., Fleming, E., et al. In-depth characterization of microRNA transcriptome in melanoma. (2013) PLoS ONE 8(9): e72699.
Online ISSN: 2381-0858

Journal Title: Investigative Dermatology and Venereology Research Journal Short Name: Invest Dermatol Venereol Res
Ommega Online Publishers

E-mail: editor.dermatology@ommegaonline.org

Website: www.ommegaonline.org 